\title{
Proof of concept studies for siRNA delivery by non-ionic surfactant vesicles: in vitro and in vivo evaluation of protein knockdown
}

\author{
Mohammad A. Obeid ${ }^{1,2 *}$, Christine Dufès ${ }^{2}$, Sukrut Somani², Alexander B. Mullen², \\ Rothwelle J. Tate $^{2}$, Valerie A. Ferro ${ }^{2}$ \\ ${ }^{1}$ Faculty of Pharmacy, Yarmouk University, Irbid, Jordan. \\ ${ }^{2}$ Strathclyde Institute of Pharmacy and Biomedical Sciences, University of Strathclyde, 161 \\ Cathedral Street, G4 0RE Glasgow, United Kingdom. \\ *Corresponding author E-mail Address: m.obeid@yu.edu.jo
}

Tel: +962798270457

\section{Abbreviations}

NISV: Non-ionic surfactant vesicles; CN: cationic niosomes; PBS: Phosphate buffered saline;

Chol: Cholesterol; T85: Tween 85; PDI: Polydispersity index; ZP: Zeta potential; siRNA: Small interfering RNA; RNAi: RNA interference; FACS: fluorescence-activated cell sorter. 


\title{
Graphical abstract
}

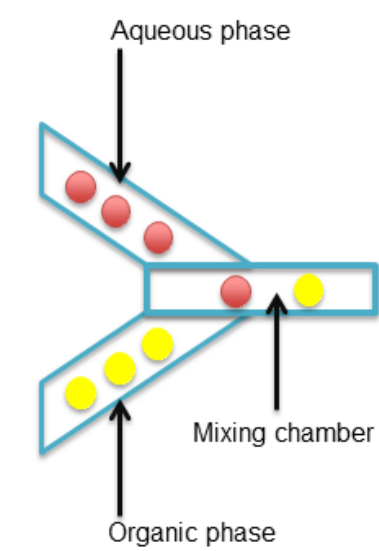

Microfluidic production
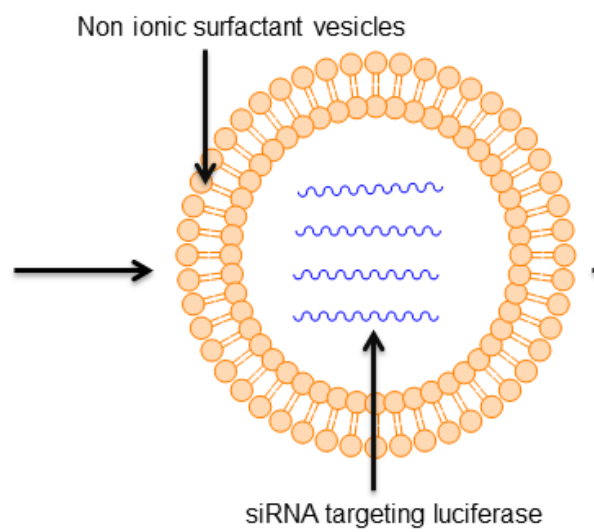

Delivery system

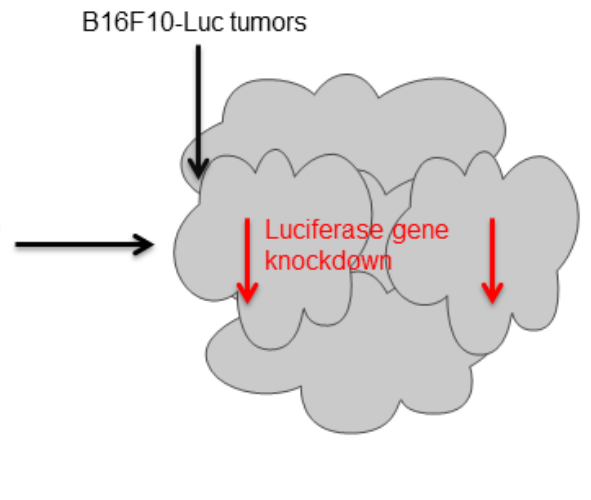

Gene Silencing

\begin{abstract}
RNA interference (RNAi) is an effective and naturally occurring post-transcriptional gene regulatory mechanism. This mechanism involves the degradation of a target messenger RNA (mRNA) through the introduction of short interfering RNA (siRNA) that is complementary to the target mRNA. The application of siRNA-based therapeutics is limited by the development of an effective delivery system, as naked siRNA is unstable and cannot penetrate the cell membrane. In this study, we investigated the use of cationic niosomes $(\mathrm{CN})$ prepared by microfluidic mixing for siRNA delivery. In an in vitro model, these vesicles were able to deliver anti-luciferase siRNA and effectively suppress luciferase expression in B16-F10 mouse melanoma cells. More importantly, in an in vivo mouse model, intratumoral administration of CN-carrying anti-luciferase siRNA led to significant suppression of luciferase expression
\end{abstract}


compared with naked siRNA. Thus, we have established a novel and effective system for the delivery of siRNA both in vitro and in vivo, which shows high potential for future application of gene therapeutics.

\section{Key Words}

Niosomes, Nanoparticles, Microfluidics, RNA interference, Drug delivery 


\section{Introduction}

Short interfering RNA (siRNA) has gained substantial interest as a promising therapeutic agent as it has the ability to silence specific upregulated genes through a RNA inhibitory (RNAi) mechanism and therefore hindering corresponding protein expression. However, the efficacy of siRNA therapy is significantly hampered by poor cellular membrane penetration, rapid degradation by RNase enzymes, non-specific tissue distribution, and short circulating time [1, 2]. Therefore, therapeutic application of siRNA requires the use of an efficient delivery vehicle that can carry, protect, and efficiently deliver siRNA into target cells [3]. In this regard, lipidbased nanoparticles, including liposomes, have been widely investigated as possible siRNA carriers because of their advantages such as high loading efficiency and biocompatibility [4, 5]. Through their membrane bilayer structure, lipid nanoparticles can protect siRNA that is embedded in the aqueous core or adsorbed on the surface of the nanoparticles [6]. Although liposomes have been demonstrated to be successful in siRNA delivery, they have limitations for widespread use such as stability and high production costs. Non-ionic surfactant vesicles (NISV, niosomes) are one type of lipid-based nanoparticle that have a membrane bilayer structure similar to liposomes [7, 8]. NISV consist of non-ionic surfactants, in addition to cholesterol and charging species in place of the phospholipids used in liposomes [9]. These non-ionic surfactants are composed of a hydrophilic head and a hydrophobic tail that will spontaneously arrange in a bilayer structure upon hydration [10]. The use of non-ionic surfactants in NISV improves the stability of these particles and decreases the production cost compared to liposomes. Cholesterol is another component in the NISV structure that modulates the mechanical strength and water permeability of the bilayer structure [11]. Other additives in NISV include charged molecules which enhance the stability of the formulated vesicles by inducing electrostatic repulsion between individual particles [9]. NISV have gained 
considerable interest as a drug delivery system and there are many successful reports for their application in cancer [12, 13], diabetes [14, 15], and transdermal drug delivery [16, 17]. Nevertheless, there are limited reports about the use of NISV as a delivery vehicle for siRNA [18], which has significant potential and needs to be investigated and developed in order to mediate efficacious gene silencing for therapeutic applications.

Previously, we have reported the in vitro efficacy of cationic niosomes $(\mathrm{CN})$ formulation in suppressing green fluorescent protein (GFP) expression in copGFP-A549 cells by anti-GFP siRNA [19]. In the present study, to confirm the gene silencing results observed on copGFPA549 cells and to further explore the in vivo efficacy, the biological activity of the $\mathrm{CN}$ formulation was tested on a different cell model using another protein reporter. B16-F10 mouse melanoma cells stably expressing luciferase enzyme were used. The $\mathrm{CN}$ formulation were loaded with either AllStars Alexa Fluor ${ }^{\circledR} 488$ (AF488)-labelled Negative Control siRNA to confirm cellular uptake of siRNA or with anti-luciferase siRNA (siLUC) to confirm the effectiveness of these formulations in delivering siRNA and suppressing luciferase enzyme expression. An in vivo experiment was then carried out to assess the luciferase suppression in an animal model.

\section{Materials and methods}

\subsection{Materials}

Polyoxyethylenesorbitan trioleate (Tween 85), cholesterol (Chol), dimethyldioctadecylammonium bromide (DDAB), resazurin powder, serum-free and antibiotic-free Roswell Park Memorial Institute medium (RPMI 1640), L-glutamine, penicillin-streptomycin were purchased from Sigma-Aldrich (Irvine, UK) (all at cell culture grade). Foetal bovine serum (FBS) was purchased from Biosera (East Sussex, UK). Sodium pyruvate $(100 \mathrm{mM})$ and minimum essential medium non-essential amino acids (MEM NEAA) 
were purchased from Life Technologies (Loughborough, UK). Mouse melanoma B16-F10luc-G5 luciferase expressing cells and D-luciferin were obtained from Caliper life Science (Hopkinton, USA). Sterile, RNase-free phosphate buffered saline 1M and sterile, RNase-free water were purchased from LONZA (Slough, UK). AllStars AF488-labelled Negative Control siRNA and HiPerFect transfecting reagent were purchased from Qiagen (Manchester, UK). The anti-luciferase siRNA (siLUC) duplex sequence (Sense: rGrArGrGrCrUrArArGrGrUrGrGrUrGrGrArCrUrUrGrGrACA, Antisense: rUrGrUrCrCrArArGrUrCrCrArCrCrArCrCrUrUrArGrCrCrUrCrGrA) were synthesised by Integrated DNA Technologies (Leuven, Belgium). ONE-Glo ${ }^{\mathrm{TM}}$ luciferase assay system was purchased from Promega Corporation (Southampton, UK).

\subsection{Formulation of the cationic niosomes $(\mathrm{CN})$}

CN composed of T85:Chol:DDAB (at a molar ratio of 40:40:20) were prepared by microfluidic mixing as described previously [20] using sterile RNase-free $5 \%(\mathrm{w} / \mathrm{v})$ glucose as an aqueous medium. Briefly, a specific volume of the aqueous medium was mixed with the lipid phase in ethanol at a volumetric flow rate of 3:1 (aqueous: lipid) in the microfluidic micromixer at a total flow rate of $12 \mathrm{~mL} /$ minute $(9 \mathrm{~mL} /$ minute for the aqueous phase and $3 \mathrm{~mL} /$ minute for the lipid phase) at $50^{\circ} \mathrm{C}$. The mixing process was carried out using a NanoAssemblr ${ }^{\mathrm{TM}}$ (Benchtop, Precision NanoSystems Inc., Vancouver, Canada).

\subsection{Characterisation of the $\mathrm{CN}$}

Particle size, polydispersity index (PDI) and zeta potential (ZP) were measured with a Zetasizer Nano-ZS (Malvern Instruments, UK). These measurements were carried out at $25^{\circ} \mathrm{C}$ at a $1 / 20$ dilution in the same medium used for the particle preparation. All samples were prepared in triplicate and the $\mathrm{Z}_{\text {Average, }}$ PDI, and $\mathrm{ZP}$ reported. 


\subsection{Cell viability assay}

To evaluate the cytotoxicity of the $\mathrm{CN}, \mathrm{B} 16-\mathrm{F} 10$ cells were seeded into a 96-well plate at a density of $1 \times 10^{4}$ per well in $100 \mu 1$ RPMI 1640 medium supplemented with $10 \%$ (v/v) FBS, 1 $\%(\mathrm{v} / \mathrm{v}) \mathrm{L}$-glutamine and $1 \%(\mathrm{v} / \mathrm{v})$ penicillin-streptomycin and incubated at $37{ }^{\circ} \mathrm{C}, 5 \% \mathrm{CO}_{2}$ and $100 \%$ humidity. Twenty-four hours later, cells were treated with $\mathrm{CN}$ at concentrations from 9.77-1250 $\mu \mathrm{g} / \mathrm{ml}$. Ten-percent dimethyl sulphoxide (DMSO) was used as positive control and untreated cells as negative control. The treated cells were incubated for a further $24 \mathrm{~h}$ and then $20 \mu \mathrm{l}$ of resazurin $(0.1 \mathrm{mg} / \mathrm{ml})$ was added to each well and incubated for a further $24 \mathrm{~h}$. The quantity of resorufin produced resulting from metabolism of resazurin by viable cells, was measured on a SpectraMax M5 plate reader (Molecular Devices, USA) at $560 \mathrm{~nm}-590 \mathrm{~nm}$. Cell viability was expressed as a percentage of the untreated control cells. The results were expressed as a mean and standard deviation obtained from three experiments.

\subsection{In vitro cellular uptake}

Cellular uptake of CN by B16-F10 cells was quantified by fluorescence-activated cell sorter (FACS). B16-F10 cells were seeded in 12 -well plates at $1 \times 10^{5}$ cells/well in $1100 \mu \mathrm{L}$ of RPMI 1640 culture media, supplemented with $10 \%(\mathrm{v} / \mathrm{v})$ FBS, 1\% (v/v) L-glutamine and 1\% (v/v) MEM NEAA (without antibiotics), $24 \mathrm{~h}$ before experiments at $37^{\circ} \mathrm{C}, 5 \% \mathrm{CO}_{2}$ and $100 \%$ humidity. CN/siRNA complexes (termed nioplexes) were prepared as follows: an appropriate volume of siRNA (from $10 \mu \mathrm{M}$ stock) was mixed with the $\mathrm{CN}$ formulation (from a $625 \mu \mathrm{g} / \mathrm{ml}$ stock) with pipetting up and down to ensure optimal mixing. The nioplex samples were incubated at room temperature for $30 \mathrm{~min}$ to allow the formation of transfection complexes.

Cells were then treated with CN encapsulating AF488-labelled Negative Control siRNA at a final concentration of $20 \mathrm{nM} /$ well. The results were compared to those obtained with the 
positive control HiPerFect transfecting reagent and the experiments were done with the use of siRNA alone, $\mathrm{CN}$ alone and untreated cells as controls. Cells were incubated for $72 \mathrm{~h}$ and then the media was removed, cells were trypsinised and diluted with PBS to $1 \mathrm{ml}$. The cell suspension was then centrifuged at $1200 \mathrm{rpm}$ for $5 \mathrm{~min}$ and then the pellet re-suspended in 1 $\mathrm{ml}$ of FACS buffer $(10 \%, \mathrm{v} / \mathrm{v}, \mathrm{FBS}$ in PBS). Quantitative cellular uptake was measured using a FACSCanto flow cytometer, BD Biosciences (UK) using FACS Diva software. Upon acquisition, the cells were gated using forward scatter versus side scatter (FCS vs SSC) to eliminate dead cells and debris. Cells $(10,000)$ were collected for each sample and the data were analysed with FACS Diva software. The results are presented as a mean and standard deviation obtained from three samples.

\subsection{In vitro luciferase gene silencing assay}

B16-F10 cells were plated in a 96-well plate (7500 cells/well) in $75 \mu$ RPMI medium containing 10\% (v/v) FBS, 1\% (v/v) L-glutamine and 1\% (v/v) MEM NEAA (without antibiotics) at $37^{\circ} \mathrm{C}, 5 \% \mathrm{CO}_{2}, 100 \%$ humidity for $24 \mathrm{~h}$ prior to transfection. Cells were then treated with $\mathrm{CN}$ or the positive control HiPerFect, containing siLUC at final concentrations of 0, 10, 25, 50, 100, and $200 \mathrm{nM}$ per well. siLUC alone (naked siLUC) and particles alone (mock transfection) were used as controls. The expressed luciferase level in the cells was measured after 24,48 , and $72 \mathrm{~h}$ of transfection using a ONE-Glo ${ }^{\mathrm{TM}}$ luciferase assay system following the manufacturer's protocol. Briefly, at each time point, $100 \mu 1$ of ONE-Glo reagent from the assay kit was added to the cells in each well and incubated at $25^{\circ} \mathrm{C}$ for $3 \mathrm{~min}$. The bioluminescence expressed in Relative Luminescence Unit (RLU) was measured using an in vivo imaging system (IVIS) (IVIS Spectrum ${ }^{\circledR}$, PerkinElmer, UK). Luciferase activity of a sample was expressed as the percentage of luminescence intensity compared to untreated cells. The percentage of luciferase expression was calculated by the equation: 


$$
\% \text { expression }=(\text { RLUluc } / \text { RLUctl }) * 100
$$

Where RLUluc is the mean of RLU for luc in treated cells and RLUctl is the mean of RLU for untreated cells. The results were reported as the mean and standard deviation of four different experiments.

\subsection{In vivo silencing study}

\subsubsection{Animals}

Female BALB/c nude mice, 42-49 days old (average weight of $20 \mathrm{~g}$ ), were purchased from Charles River Laboratories (UK). Mice were housed in groups of three at $19^{\circ} \mathrm{C}$ to $23^{\circ} \mathrm{C}$ with a 12-h light-dark cycle. They were fed a conventional diet (Rat and Mouse Standard Expanded, B\&K Universal, UK), with mains water provided ad libitum. The in vivo experiments described below were performed in accordance with the UK Home Office regulations.

\subsubsection{Determination of the most tolerable dose of $\mathrm{CN} / \mathrm{siLUC}$}

Two female BALB/c nude mice were injected intraperitoneally (i.p.) every day respectively with increased concentrations of empty nanoparticles $(0.2 \mathrm{ml}$, starting from $39 \mu \mathrm{g} / \mathrm{ml}$ nanoparticles) and siLUC nanoparticles $(0.2 \mathrm{ml}$, from $625 \mathrm{nM} \mathrm{siLUC})$ in order to determine the maximum tolerable dose of formulations. The mice were monitored daily for any changes in body weight as a surrogate marker of toxicity.

\subsubsection{In vivo luciferase gene silencing study}

BALB/c female nude mice were injected subcutaneously with $1 \times 10^{6}$ B16-F10-Luc cells. Seven days later, when the tumours became vascularised and palpable, a single dose of siLUC (625 $\mathrm{nM})$ loaded in $\mathrm{CN}(0.2 \mathrm{ml}$, at $39 \mu \mathrm{g} / \mathrm{ml})$ was injected intratumorally. Naked siLUC, particles alone, and untreated mice were used as controls. Three mice were used in each group. The light emitted as a result of luciferase gene expression was visualised in mice using quantitative whole-body imaging [21]. To this end, mice received an i.p. injection of the luciferase substrate 
D-luciferin $(150 \mathrm{mg} / \mathrm{kg}$ body weight) after $4,12,24$, and $48 \mathrm{~h}$ of treatment and were anesthetised by isoflurane inhalation. Ten min post-injection, bioluminescence was measured for 2 min using the IVIS Spectrum ${ }^{\circledR}$. Data were analysed using Living Image ${ }^{\circledR}$ software (PerkinElmer, UK). The resulting pseudo-colour images represent the luciferase expression within the animal. Identical illumination settings were used for acquiring all images. Luciferase expression in the treated mice was expressed as a percentage of luminescence intensity compared to the untreated mice.

\subsection{Statistical analysis}

Results were expressed as means \pm standard deviation of three readings. Statistical significance was assessed by one-way analysis of variance (ANOVA) and Tukey multiple comparison test and t-test was performed for paired comparisons using Minitab ${ }^{\circledR}$ software, State College, PE. Differences were considered statistically significant for $p$ values $<0.05$.

\section{Results}

\subsection{CN characterisation}

$\mathrm{CN}$ prepared by microfluidic mixing, using sterile RNase-free $5 \%(\mathrm{w} / \mathrm{v})$ glucose for particles preparation, were characterised by DLS. The average particles size was $61.37 \pm 0.16 \mathrm{~nm}$ with a PDI value of $0.18 \pm 0.01$ indicating homogeneous distribution. Moreover, the average ZP was $55.80 \pm 6.55 \mathrm{mV}$. Further physicochemical properties were reported previously [19].

\subsection{Cell viability assay}

Toxicity of the $\mathrm{CN}$ was assessed on B16-F10 cells using various concentrations of CN (9.77$1250 \mu \mathrm{g} / \mathrm{ml}$ ) to quantify cell viability and determine the concentration that caused $50 \%$ cell death $\left(\mathrm{EC}_{50}\right)$. Figure 1 shows the dose-response curves for the cells treated with the $\mathrm{CN}$ and the calculated $\mathrm{EC}_{50}$. The cell viability decreased significantly by increasing the $\mathrm{CN}$ concentration especially at concentrations of $312.5 \mu \mathrm{g} / \mathrm{ml}$ and above where the cells showed 
minimal viability. The $\mathrm{EC}_{50}$ value was $84.03 \pm 7.39 \mu \mathrm{g} / \mathrm{ml}$. However, the $\mathrm{CN}$ formulation was not toxic at or below $40 \mu \mathrm{g} / \mathrm{ml}$ in which the cells were $100 \%$ viable. Therefore, all subsequent experiments that included siRNA transfection were carried out so the final NISV concentration was below $40 \mu \mathrm{g} / \mathrm{ml}$.

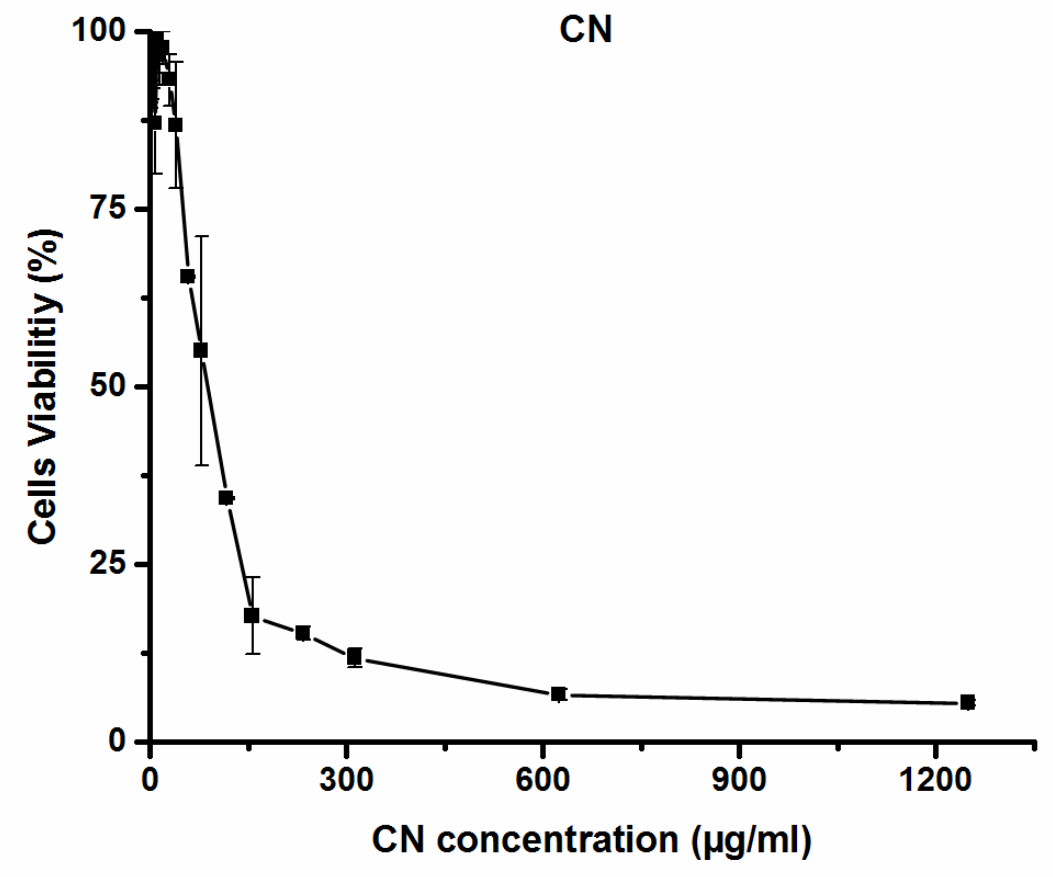

3.3.

Figure 1 Cytotoxicity of the CN Formulation on B16-F10 cells. The data represents the mean $\pm \mathrm{SD}(\mathrm{n}=3)$.

\section{B16-F10 cellular uptake}

To evaluate the cellular uptake, B16-F10 mouse melanoma cells were treated with $\mathrm{CN}$ formulation loaded with AF488-labelled negative control siRNA. The treated cells were analysed by FACS for quantitative cellular uptake (Figure 2). As can be seen in Figure 2A, B16-F10 cells did not show any siRNA uptake after being treated with siRNA alone, which can be confirmed by the very low MFI (Figure 2B) and the histogram curve (Figure 2C) compared to untreated cells. The cellular uptake for cells treated with AF488-labelled negative 
control siRNA encapsulated in the $\mathrm{CN}$ formulation was $88.28 \pm 2.29 \%$ (Figure 2A). This cellular uptake was significantly $(\mathrm{p}<0.05)$ higher than the cellular uptake achieved by HiPerFect $(70.77 \pm 4.35 \%)$ and the MFI was significantly higher for cells treated with AF488labelled negative control siRNA encapsulated by $\mathrm{CN}$ or HiPerFect compared to naked siRNA (Figure 2B). When the $\mathrm{CN}$ formulation alone without AF488-labelled negative control was used, the MFI values were low, indicating no auto-fluorescence (Figure 2B) and the histogram curves had slightly shifted compared to untreated cells (Figure 2C). 
A

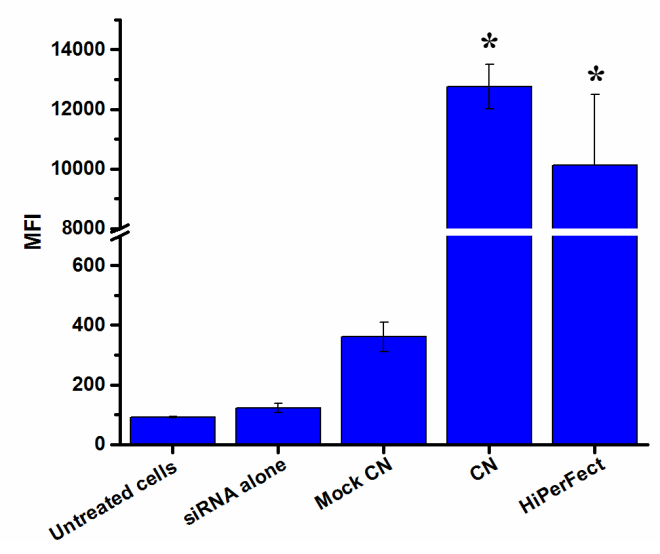

B

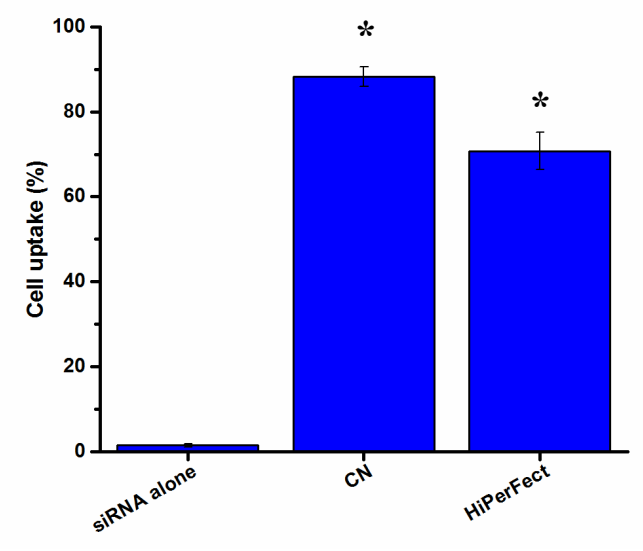

C
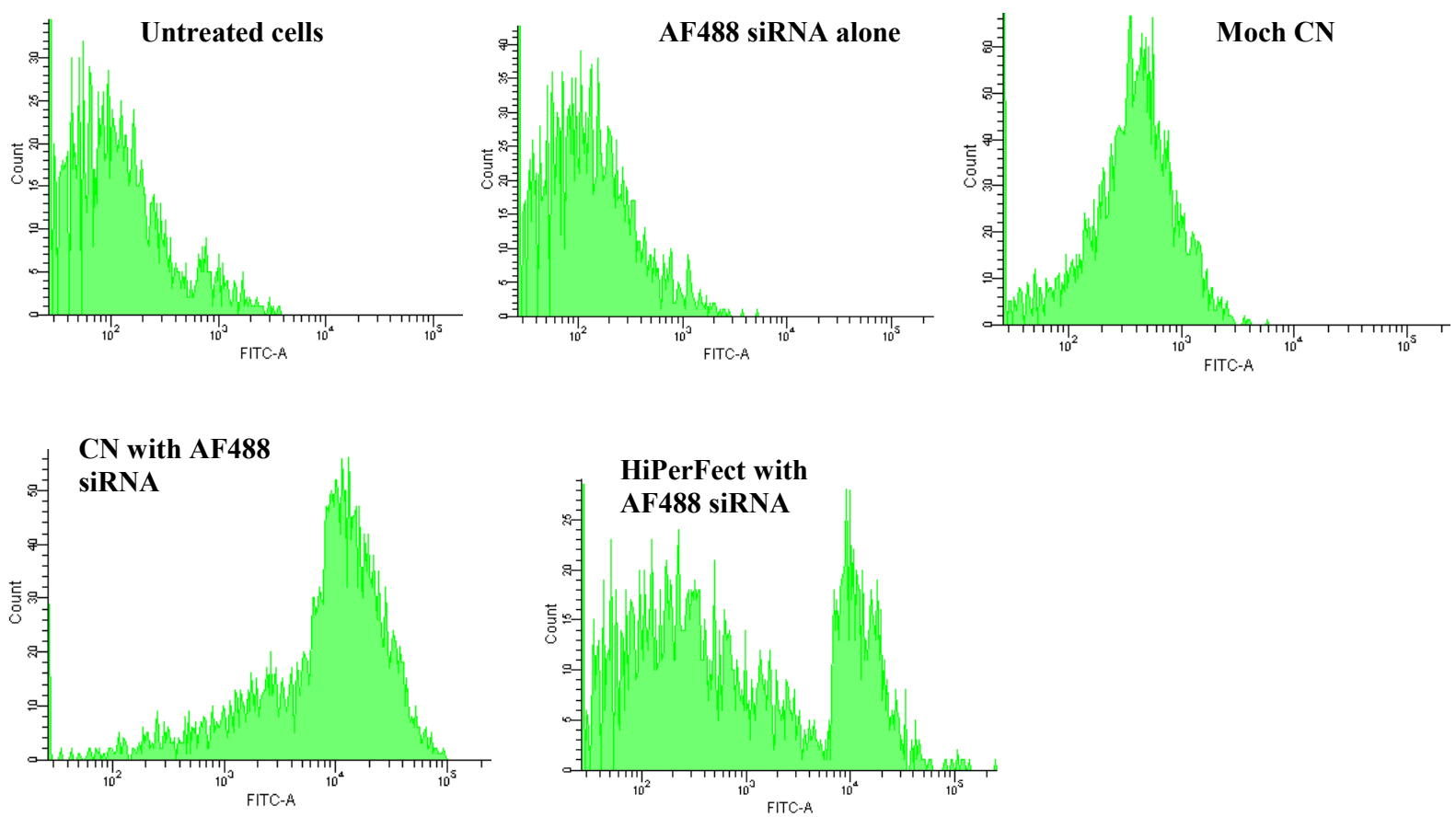

Figure 2 FACS results for (A) MFI, (B) percentages of cellular uptake, and (C) flow cytometry histograms of B16-F10 cellular uptake when treated with $\mathrm{CN}$ or HiPerFect loaded with AF488-labelled negative control siRNA. Images are representative of three independent images from each sample. The data represents means \pm standard deviation $(n=3) .{ }^{*} \mathrm{p}<0.05$ significant difference from cells treated with naked siRNA. CN: cationic niosomes, Moch CN: empty cationic niosomes. 


\subsection{In vitro luciferase gene silencing study}

We examined the extent of gene silencing by siLUC loaded in the $\mathrm{CN}$ formulation. To evaluate the gene knockdown efficiencies, B16-F10 cells stably expressing luciferase were incubated with CN formulation, loaded with various concentrations of luc siRNA, for 24, 48, and $72 \mathrm{~h}$. The specificity of the siLUC was confirmed using scrambled negative control siRNA. Luciferase expression was measured by quantifying the luciferase luminescence intensity at each time point in the cells treated with $\mathrm{CN}$ loaded with various concentrations of siLUC (Figure 3). The luciferase enzyme knockdown was compared to untreated cells. CN demonstrated significant $(\mathrm{p}<0.05)$ transfection efficiency, which was concentration- and timedependent. After $24 \mathrm{~h}$ incubation, the percentage of luciferase expression (compared to untreated cells) decreased significantly $(\mathrm{p}<0.05)$ from $77.01 \pm 2.22 \%$ to $54.42 \pm 2.06 \%$ by increasing the siLUC concentration from $10-200 \mathrm{nM}$. After $48 \mathrm{~h}$ incubation, all the siLUC concentrations induced the same level of luciferase expression inhibition resulting in a luciferase expression of around 30\% (Figure 3). Longer incubation times with CN formulation did not result in higher luciferase inhibition. This significant luciferase inhibition, achieved by the $\mathrm{CN}$ formulation, was much higher than the inhibition demonstrated by HiPerFect. With HiPerFect, the luciferase inhibition was dose-dependent at all time points with the highest knockdown achieved after 48 and $72 \mathrm{~h}$ with no significant difference between both time points. After $24 \mathrm{~h}$ incubation, the highest luciferase inhibition achieved when the cells were transfected with $200 \mathrm{nM}$ siLUC using HiPerFect was $75.54 \pm 0.56 \%$ luciferase expression. This was significantly $(\mathrm{p}<0.05)$ higher than the luciferase expression when the cells were treated with the $\mathrm{CN}$ formulation using the same siLUC concentration (luciferase expression $54.42 \pm 2.06 \%$ ). Longer incubation time for cells transfected with HiPerFect resulted in higher luciferase inhibition, where the maximum effect seen after $48 \mathrm{~h}$ using $200 \mathrm{nM}$ of siLUC resulted in luciferase expression of $48.35 \pm 4.48 \%$ with no further effect at longer incubation times (Figure 
3). No effect was noticed for $\mathrm{CN}$ formulation at $0 \mathrm{nM}$ siLUC (mock transfection) at all time points. Similarly, no effect was seen when using siLUC alone at all time points (data not shown). This indicates that the decrease in luciferase expression using siLUC was indeed caused by the sequence specific gene silencing of siLUC.
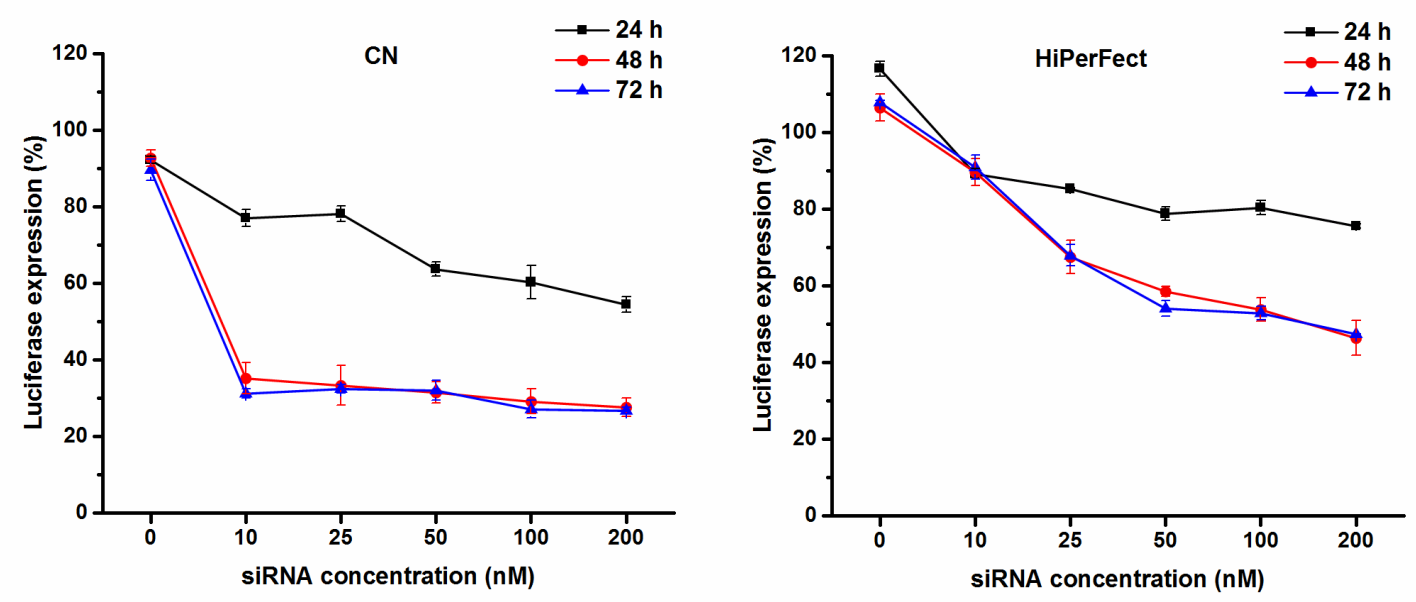

Figure 3 Percentage of luciferase expression in B16-F10 cells after being transfected by various siLUC concentrations using $\mathrm{CN}$ formulation and HiPerFect. Luminescence was measured after 24,48 , and $72 \mathrm{~h}$ of incubation. Data represents mean $\pm S D(n=3)$.

\subsection{In vivo silencing study}

\subsubsection{Determination of the most tolerable dose of CN/siLUC}

In a pilot study, empty particles at a concentration of $39 \mu \mathrm{g} / \mathrm{mL}$ was found to have no effect on the weight of the animal, whereas at concentrations higher than $156 \mu \mathrm{g} / \mathrm{mL}$, there was a decrease in weight (Table 1). This concentration of empty particles was therefore selected and used to encapsulate various concentrations of siLUC. A siLUC concentration at $625 \mathrm{nM}$ was found to be well tolerated by the mouse while concentrations higher than $1250 \mathrm{nM}$ caused 
weight loss (Table 2). Therefore, in the following in vivo experiments, CN/siLUC nioplexes were prepared using $39 \mu \mathrm{g} / \mathrm{ml}$ of $\mathrm{CN}$ encapsulating $625 \mathrm{nM}$ of siLUC.

Table 1 Change of the mouse weight when given increasing doses of empty particles of CN.

\begin{tabular}{|c|c|c|c|c|c|}
\hline \multicolumn{7}{|c|}{ Empty particles } \\
\hline Days & 1 & 2 & 3 & 4 & 5 \\
\hline Particle concentration ( $\boldsymbol{\mu g} / \mathbf{m l})$ & 39 & 156 & 315.5 & - & - \\
\hline siRNA concentration (nM) & 0 & 0 & 0 & - & - \\
\hline Animal weight (g) & 19.2 & 19.3 & 18.7 & 18.6 & 18.4 \\
\hline
\end{tabular}

Table 2. Change of the mouse weight when given increasing doses of siLUC encapsulated in $\mathrm{CN}$ at concentration of $39 \mu \mathrm{g} / \mathrm{ml}$.

\begin{tabular}{|c|c|c|c|c|c|}
\hline \multicolumn{7}{|c|}{ Particles + siLUC } \\
\hline Days & 1 & 2 & 3 & 4 & 5 \\
\hline Particle concentration ( $\mu \mathrm{g} / \mathbf{m l})$ & 39 & 39 & 39 & - & - \\
\hline siRNA concentration (nM) & 625 & 1250 & 2500 & - & - \\
\hline Animal weight (g) & 19 & 19.3 & 18.8 & 18.6 & 18.8 \\
\hline
\end{tabular}

\subsubsection{In vivo luciferase gene silencing study}

To investigate whether $\mathrm{CN}$ could release its encapsulated siRNA and inhibit gene expression in tumours, $\mathrm{CN}$ loaded with siLUC $(39 \mu \mathrm{g} / \mathrm{ml}$ of $\mathrm{CN}$ encapsulating $625 \mathrm{nM}$ of siLUC) were injected intratumorally in nude mice bearing subcutaneous B16-F10-Luc melanoma. Luciferase expression in the tumour was evaluated qualitatively and quantitatively in 
anaesthetised animals. Mice injected with siLUC alone, $\mathrm{CN}$ alone, or left untreated were used as controls. Figure 4 shows the average bioluminescence measured for each group. A representative mouse whose emitted light was closest to the average for that group ( 3 mice per group) is shown (Figure 5).

Luciferase expression in mice injected with siLUC encapsulated in $\mathrm{CN}$ was significantly (p $<0.05$ ) decreased, $4 \mathrm{~h}$ after injection, by about $50 \%$ (Figure 4 ). The maximum luciferase expression knockdown (by 70\%) was obtained $12 \mathrm{~h}$ after injection. This inhibition was reversible and the luciferase expression returned to normal after $24 \mathrm{~h}$. In contrast, the bioluminescence signals in the mice treated with naked siLUC increased over the same time period, suggesting no inhibition of luciferase expression at any time point. Luciferase expression did not appear to be affected by the $\mathrm{CN}$ formulation, as the bioluminescence signal increased over time following treatment with empty $\mathrm{CN}$. These results suggest that the inhibition of luciferase expression resulted from siLUC delivery by the $\mathrm{CN}$ into the cytoplasm of the cells and the subsequent RNAi mechanism. This demonstrates that siRNA can be released from the $\mathrm{CN}$ to the cancer cells and can inhibit target gene expression in vivo. 

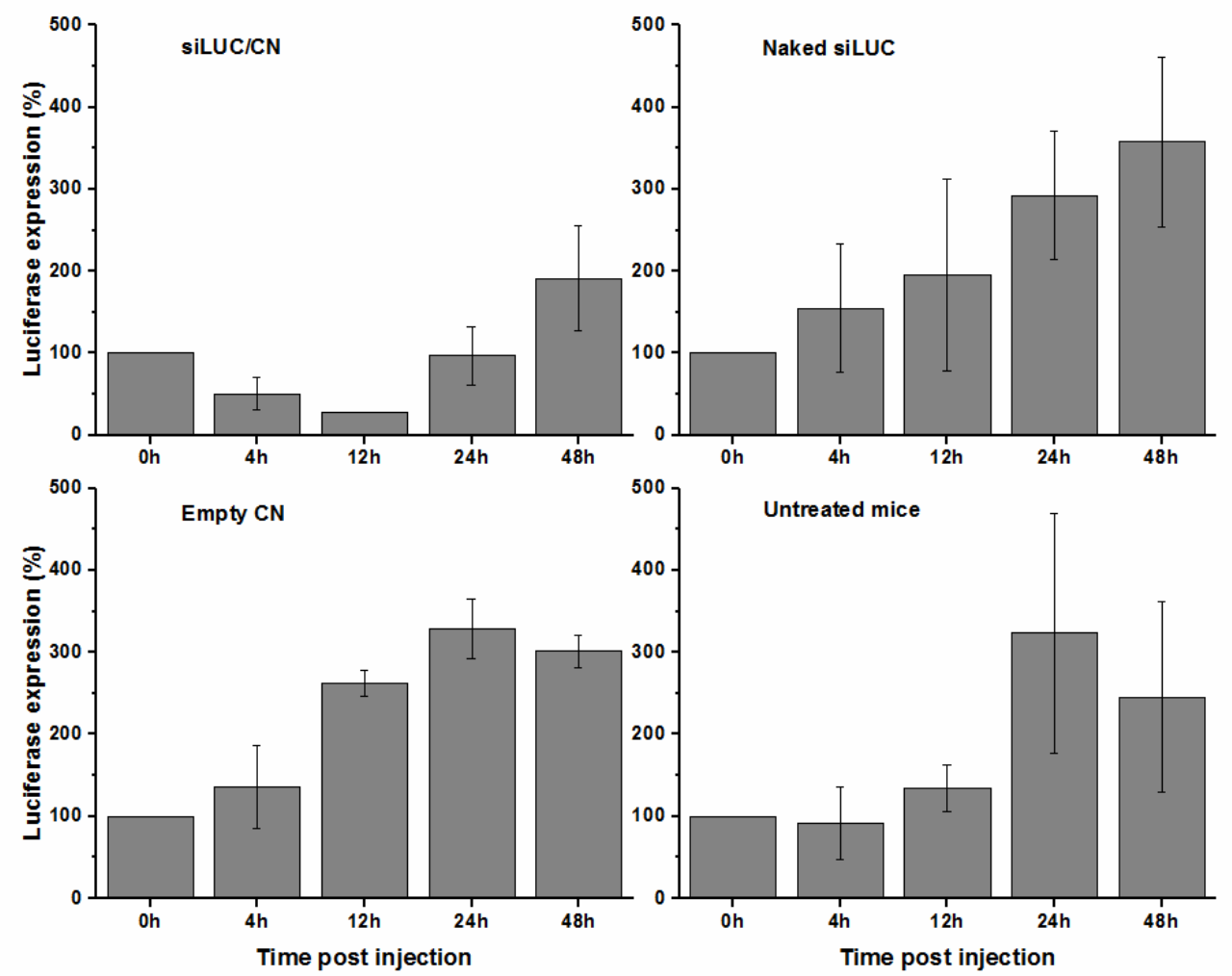

Figure 4 Bioluminescence of mice injected with siLUC/CN nioplexes, naked siLUC, empty $C N$, or left untreated. Readings were taken at $t=0 \mathrm{~h}, 4 \mathrm{~h}, 12 \mathrm{~h}, 24 \mathrm{~h}$, and $48 \mathrm{~h}$ post intra-tumour injection. Results represent the average of readings taken from three mice in each group \pm SD. 

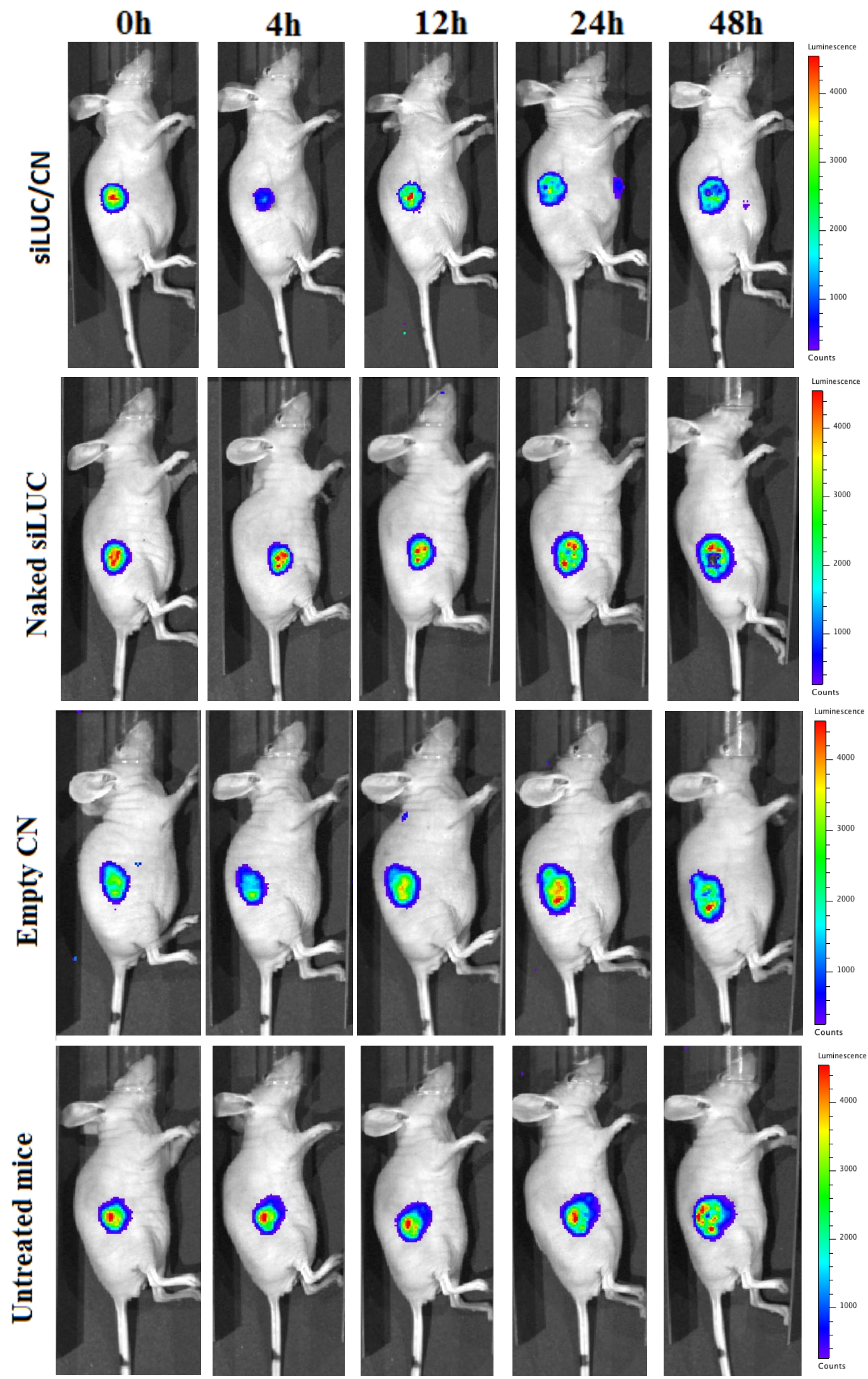

Figure 5 IVIS images of mice receiving siLUC/CN, naked siLUC, empty CN, and untreated mice at zero time and after $4,12,24$, and 48 hours post intra-tumour injection. A representative mouse whose emitted light was closest to the average for that groun ( 3 mice ner groun) is shown. 


\section{Discussion}

Numerous barriers have to be overcome for the effective delivery of siRNA into the cytoplasm. The main obstacle to the development of effective therapeutics with siRNA is a suitable delivery system. First, the delivery system has to remain stable over time, to be of minimal toxicity, to be taken up by the cells, and to escape the endosome compartment with the subsequent delivery of the siRNA for its interaction with the RNAi machinery in the cytoplasm [22]. NISV possess attractive properties as a drug delivery system such as biodegradability, biocompatibility, stability, and ease of manufacture. Limited research is available about the use of NISV for siRNA delivery. Previous work for siRNA delivery combined non-ionic surfactant with phospholipids in a formulation called spanosomes [23]. In our previous study, effective various $\mathrm{CN}$ formulations were prepared and their efficacy in siRNA transfection and GFP inhibition in copGFP-A549 cells evaluated [19]. In order to confirm the gene silencing observed in copGFP-A549 cells, one formulation was taken forward in this study and the biological activity of the selected $\mathrm{CN}$ was tested on a different cell model, the B16-F10 cells, which could then be investigated in vivo in a rat model. CN cytotoxicity was evaluated on B16F10 cells to make sure that any observed luciferase knock-down was a result of the siRNA used rather than as a result of any vesicle-related toxicity. Cytotoxicity results revealed that $\mathrm{CN}$ were not toxic at or below $40 \mu \mathrm{g} / \mathrm{ml}$. Next, the transfection efficiency of the $\mathrm{CN}$ was examined by analysing the B16-F10 cellular uptake with FACS after treating these cells with $\mathrm{CN}$ encapsulating AllStars AF488-labelled Negative Control siRNA. CN were able to deliver siRNA as confirmed by the high cellular uptake percentages. This high cellular uptake can be attributed to the positive charge on the surface of $\mathrm{CN}$, which enhanced the interaction with the negatively charged cellular membrane. The cellular uptake using $\mathrm{CN}$ was compared with HiPerFect transfection reagent. Cells treated with the empty $\mathrm{CN}$ or naked siRNA showed no 
fluorescence signal, indicating that the above mentioned cellular uptake was a result of the siRNA delivery by the $\mathrm{CN}$ formulation.

After evaluating cellular uptake, luciferase gene silencing mediated by siLUC loaded in the $\mathrm{CN}$ formulation was evaluated. Luciferase is a bioluminescence producing enzyme widely used for monitoring siRNA delivering efficacy by monitoring bioluminescence changes after antiluciferase siRNA treatment $[24,25]$. After evaluating cellular uptake mediated by the $\mathrm{CN}$ formulation, the endosome release after uptake and the subsequent inhibition of the target luciferase enzyme need to be proven. To examine both the extent of gene silencing and the optimal incubation time, B16-F10 cells were transfected by various siLUC concentrations (10$200 \mathrm{nM}$ ) using the $\mathrm{CN}$ formulation and the changes in bioluminescence intensity was monitored at various time points. Naked siLUC showed negligible gene silencing effects when compared to siLUC with CN. Results indicate that the time of incubation and the siRNA concentrations had effects on the degree of gene knockdown. Increasing the incubation time meant higher exposure of the particles to the cells in order to increase the cellular uptake. Here, the efficacy rose by increasing the incubation time until $48 \mathrm{~h}$, where further incubation did not result in greater knockdown for all siRNA concentrations. This suggests that the observed downregulation effect as a result of siLUC delivery by $\mathrm{CN}$ was stable for at least $72 \mathrm{~h}$. Moreover, these experiments were carried out in the presence of serum, which indicates that the $\mathrm{CN}$ were able to protect the siLUC from degradation in the presence of serum proteins. Together, these results demonstrate that by using the $\mathrm{CN}$ formulation, siLUC is protected against degradation, internalised by the cells, and enabled escape of the endosomes to the cytoplasm where bioactivity was displayed.

$\mathrm{CN}$ were able to induce high luciferase suppression by about $68 \%$ after $48 \mathrm{~h}$ incubation using $10 \mathrm{nM}$ siLUC. CN appeared to be an effective transfection reagent when compared with the commercially available HiPerFect, which exhibited a clear dose-dependent effect on 
knockdown efficacy across all the siRNA concentrations. These results for luciferase suppression were consistent with the results reported previously on inhibition of GFP expression in A549 cells by siGFP delivered by various CN formulations [19]. This high luciferase suppression by siLUC delivered by this particular $\mathrm{CN}$ can be explained by the $\mathrm{CN}$ endosomal escape ability after uptake to the cytoplasm where the RNAi mechanism occurs [26]. $\mathrm{CN}$ were able to escape the endosome at a high rate, release the siLUC into the cytoplasm, initiating luciferase RNA interference, and inhibit the luciferase expression. This ability is due in part to the presence of T85 as a non-ionic surfactant in the $\mathrm{CN}$ formulation. T85 is believed to have fusogenic properties that enhances endosome escape by promoting instability in the endosome compartment and therefore releasing siRNA into the cytosol [27, 28]. The possibility of the downregulation of gene expression being due to cytotoxic effects of the formulations can be excluded as the non-toxic concentration of $\mathrm{CN}$ formulation was used. In addition, no luciferase downregulation was observed when cells were transfected with empty particles of $\mathrm{CN}$ alone (0 $\mathrm{nM}$ siLUC).

High siRNA concentrations can result in off-target effects which is one of the side effects associated with siRNA therapeutics [29]. Previous reports of siRNA delivery systems targeting luciferase, were able to achieve high luciferase suppression only at high siRNA concentrations, which increases the possibility of siRNA off-target effects. For example, Takemoto et al. were able to achieve $80 \%$ luciferase silencing with $100 \mathrm{nM}$ siRNA using a siRNA-grafted polymer delivery system [30]. With chitosan nanoparticles, Ragelle et al. were able to achieve the maximum of $71 \%$ luciferase suppression using $200 \mathrm{nM}$ of anti-luciferase siRNA [31]. Li et al. were able to induce around $70 \%$ luciferase gene silencing using targeted cationic liposomes using $250 \mathrm{nM}$ anti-luciferase siRNA [32]. In our CN study, comparable high luciferase silencing effects were achieved with a much lower concentration of siRNA at $10 \mathrm{nM}$, 
highlighting the efficiency of this $\mathrm{CN}$ formulation. Based on the in vitro results, the use of $\mathrm{CN}$ for in vivo RNA therapeutic applications was then evaluated.

After determining the maximum dose that could be used for the in vivo experiments, mice bearing luciferase-expressing tumours were intratumorally injected with siLUC encapsulated in $\mathrm{CN}$. After 4h, the luciferase expression decreased by about $50.77 \pm 20.35 \%$, indicating that the nioplexes were taken up by the cells where the siLUC released into the cytoplasm and incorporated in the RNA induced silencing complex (RISC) followed by luciferase expression knockdown. Twelve hours after the treatment, luciferase expression was significantly decreased by more than $70 \%$. This luciferase suppression was reversible and, $24 \mathrm{~h}$ after injection, the luciferase expression was fully recovered. This is in agreement with what has been reported in the literature about the reversibility of the RNAi mechanism [33, 34]. These results provide an insight into the possible required dosing intervals to maintain the target gene suppression by siRNA in a therapeutic application. In mice injected with naked siLUC, there was no luciferase suppression and the luciferase expression increased with time as the tumour size naturally increased, suggesting that the tumour cells did not take up naked siRNA due to their hydrophilic properties [35]. Moreover, mice injected with particles alone showed an increase in the luciferase expression over time, suggesting that the empty particles had no effect on both luciferase expression and tumour growth.

In the work of Minakuchi et al., significant luciferase suppression was achieved with an atelocollagen delivery system using a single injection via the same route of administration and the same tumour type that was used in this study [36]. However, despite the larger doses used by them, based on the tumour size $\left(2.5 \mu \mathrm{g} \operatorname{siRNA} / 50 \mu \mathrm{l} / 50 \mathrm{~mm}^{3}\right.$ tumor $)$, the luciferase expression was also reversible after 2-3 days [36]. Filleur et al. investigated the use of naked siRNA to suppress luciferase expression by intra-tumoral injection and ended up with negative 
results, which was similar to the results reported here in which naked siLUC did not induce any gene suppression [37].

These in vivo results demonstrate the efficacy of $\mathrm{CN}$ in delivering and releasing siRNA into tumour cells. Although further experiments are required such as i.v. treatment and biodistribution studies, $\mathrm{CN}$-mediated siRNA delivery possess the potential for in vivo delivery of siRNA into tumour tissues. These results are proof of concept of the ability of $\mathrm{CN}$ to effectively deliver siRNA into mouse melanoma cells. These $\mathrm{CN}$ formulations can be explored with different types of cancer cells in the expectation of similar outcomes. Moreover, these intratumor injection results could be used as a model for localised treatment of siRNA therapeutics delivered by $\mathrm{CN}$ and these formulations can also be explored further for topical applications in treatment of different diseases.

\section{Conclusions}

We successfully formulated $\mathrm{CN}$ nanoparticles that could act as an effective siRNA delivery system to deliver siRNA and suppress luciferase expression both in vitro and in vivo. With these $\mathrm{CN}$ formulations, the suppression of over-expressed genes in different cancer types can be investigated through siRNA delivery. We were able to achieve more than $70 \%$ of luciferase knockdown through $\mathrm{CN}$ both in vitro and in vivo, which is a promising delivery system in the field of nucleic acids delivery. In conclusion, we have developed CN to efficiently and safely deliver siRNA to tumour cells and demonstrated specific inhibition of luciferase gene expression. To our knowledge, our results present the first evidence that combine in vitro and in vivo gene silencing data of siRNA delivery by NISV. This suggests that NISV might be used for therapeutic application of siRNA-based therapeutics in cancer treatment. 


\section{Acknowledgments}

The authors would like to acknowledge the Jordanian Ministry of Higher Education and

Scientific Research and Yarmouk University in Jordan for funding this work.

\section{Conflict of Interest}

The authors confirm that there is no conflict of interest with this manuscript.

\section{References}

1. Ghildiyal, M. and P.D. Zamore, Small silencing RNAs: an expanding universe. Nature Reviews Genetics, 2009. 10(2): p. 94-108.

2. Siomi, M.C., Short interfering RNA-mediated gene silencing; towards successful application in human patients. Advanced drug delivery reviews, 2009. 61(9): p. 668-671.

3. Resnier, P., et al., A review of the current status of siRNA nanomedicines in the treatment of cancer. Biomaterials, 2013. 34(27): p. 6429-6443.

4. Obeid, M.A., et al., Lipid-based nanoparticles for cancer treatment, in Lipid Nanocarriers for Drug Targeting. 2018, Elsevier. p. 313-359.

5. Al Qaraghuli, M.M., et al., Where traditional drug discovery meets modern technology in the quest for new drugs. Annals of Pharmacology and Pharmaceutics, 2017. 2(11): p. 1-5.

6. Schroeder, A., et al., Lipid-based nanotherapeutics for siRNA delivery. Journal of internal medicine, 2010. 267(1): p. 9-21. 
7. Obeid, M.A., et al., The effects of hydration media on the characteristics of non-ionic surfactant vesicles (NISV) prepared by microfluidics. International Journal of Pharmaceutics, 2016.

8. Obeid, M.A., et al., Delivering natural products and biotherapeutics to improve drug efficacy. Therapeutic delivery, 2017. 8(11): p. 947-956.

9. Marianecci, C., et al., Niosomes from 80s to present: the state of the art. Advances in colloid and interface science, 2014. 205: p. 187-206.

10. Moghassemi, S. and A. Hadjizadeh, Nano-niosomes as nanoscale drug delivery systems: an illustrated review. Journal of Controlled Release, 2014. 185: p. 22-36.

11. Pozzi, D., et al., Effect of cholesterol on the formation and hydration behavior of solidsupported niosomal membranes. Langmuir, 2009. 26(4): p. 2268-2273.

12. Tavano, L., et al., Doxorubicin loaded magneto-niosomes for targeted drug delivery. Colloids and Surfaces B: Biointerfaces, 2013. 102: p. 803-807.

13. Pawar, S. and P. Vavia, Glucosamine anchored cancer targeted nano-vesicular drug delivery system of doxorubicin. Journal of drug targeting, 2016. 24(1): p. 68-79.

14. Huang, Y., et al., PEGylated synthetic surfactant vesicles (Niosomes): novel carriers for oligonucleotides. Journal of Materials Science: Materials in Medicine, 2008. 19(2): p. 607-614.

15. Pardakhty, A., J. Varshosaz, and A. Rouholamini, In vitro study of polyoxyethylene alkyl ether niosomes for delivery of insulin. International journal of pharmaceutics, 2007. 328(2): p. 130141.

16. Manosroi, A., et al., In vitro and in vivo skin anti-aging evaluation of gel containing niosomes loaded with a semi-purified fraction containing gallic acid from Terminalia chebula galls. Pharmaceutical biology, 2011. 49(11): p. 1190-1203.

17. Rungphanichkul, N., et al., Preparation of curcuminoid niosomes for enhancement of skin permeation. Die Pharmazie-An International Journal of Pharmaceutical Sciences, 2011. 66(8): p. 570-575.

18. Paecharoenchai, O., et al., Nonionic surfactant vesicles for delivery of RNAi therapeutics. Nanomedicine, 2013. 8(11): p. 1865-1873.

19. Obeid, M.A., et al., Formulation of non-ionic surfactant vesicles (NISV) prepared by microfluidics for therapeutic delivery of siRNA into cancer cells. Molecular Pharmaceutics, 2017.

20. Obeid, M.A., et al., Comparison of the Physical Characteristics of Monodisperse Non-ionic Surfactant Vesicles (NISV) Prepared Using Different Manufacturing Methods. International Journal of Pharmaceutics, 2017.

21. Contag, C.H., et al., Visualizing gene expression in living mammals using a bioluminescent reporter. Photochemistry and photobiology, 1997. 66(4): p. 523-531.

22. Khalil, I.A., et al., Uptake pathways and subsequent intracellular trafficking in nonviral gene delivery. Pharmacological reviews, 2006. 58(1): p. 32-45.

23. Zhou, C., et al., SPANosomes as delivery vehicles for small interfering RNA (siRNA). Molecular pharmaceutics, 2011. 9(2): p. 201-210.

24. Fan, F. and K.V. Wood, Bioluminescent assays for high-throughput screening. Assay and drug development technologies, 2007. 5(1): p. 127-136.

25. Reynolds, A., et al., Rational siRNA design for RNA interference. Nature biotechnology, 2004. 22(3): p. 326.

26. Pozzi, D., et al., Mechanistic evaluation of the transfection barriers involved in lipid-mediated gene delivery: interplay between nanostructure and composition. Biochimica et Biophysica Acta (BBA)-Biomembranes, 2014. 1838(3): p. 957-967.

27. Huang, Y., et al., Polysorbate cationic synthetic vesicle for gene delivery. Journal of Biomedical Materials Research Part A, 2011. 96(3): p. 513-519.

28. Koltover, I., et al., An inverted hexagonal phase of cationic liposome-DNA complexes related to DNA release and delivery. Science, 1998. 281(5373): p. 78-81. 
29. Jackson, A.L. and P.S. Linsley, Recognizing and avoiding siRNA off-target effects for target identification and therapeutic application. Nature reviews Drug discovery, 2010. 9(1): p. 5767.

30. Takemoto, H., et al., Polyion complex stability and gene silencing efficiency with a siRNAgrafted polymer delivery system. Biomaterials, 2010. 31(31): p. 8097-8105.

31. Ragelle, H., et al., Chitosan nanoparticles for siRNA delivery: optimizing formulation to increase stability and efficiency. Journal of Controlled Release, 2014. 176: p. 54-63.

32. Li, S.-D., S. Chono, and L. Huang, Efficient gene silencing in metastatic tumor by siRNA formulated in surface-modified nanoparticles. Journal of Controlled Release, 2008. 126(1): p. 77-84.

33. Dickins, R.A., et al., Tissue-specific and reversible RNA interference in transgenic mice. Nature genetics, 2007. 39(7): p. 914-921.

34. Kanasty, R., et al., Delivery materials for siRNA therapeutics. Nature materials, 2013. 12(11): p. 967-977.

35. Whitehead, K.A., R. Langer, and D.G. Anderson, Knocking down barriers: advances in siRNA delivery. Nature reviews Drug discovery, 2009. 8(2): p. 129-138.

36. Minakuchi, Y., et al., Atelocollagen-mediated synthetic small interfering RNA delivery for effective gene silencing in vitro and in vivo. Nucleic acids research, 2004. 32(13): p. e109-e109.

37. Filleur, S., et al., SiRNA-mediated inhibition of vascular endothelial growth factor severely limits tumor resistance to antiangiogenic thrombospondin-1 and slows tumor vascularization and growth. Cancer research, 2003. 63(14): p. 3919-3922. 